\section{Prevalencia de trastornos psiquiátricos en adolescentes infractores de ley en Chile}

\author{
JORGE GAETE ${ }^{1}$, NICOLÁS LABBÉ ${ }^{1, a}$, PALOMA DEL VILLAR ${ }^{2, b}$, \\ CATALINA ALLENDE ${ }^{2, b}$, EDUARDO VALENZUELA ${ }^{2, b}$
}

\section{Prevalence of psychiatric disorders among teenage offenders}

Background: There has been a significant number of adolescents admitted to National Youth Service of Chile (SENAME) detention centers in recent years, specifically since the promulgation of a law reform on juveniles' penal responsibility for people aged 14 to 18 years (2006). Aim: To determine the prevalence of psychiatric disorders in adolescent male offenders aged between 14 and 17 years. Material and Methods: The Mini International Neuropsychiatric Interview for Kids and Adolescents (MINI-KID) was applied to 489 adolescents admitted to detention centers, to determine the presence of psychiatric disorders. (Hypo) maniac episodes, eating disorders, pervasive developmental disorders, tic disorders, and psychotic disorders were excluded. Furthermore, for purposes of analysis, 23 cases that used a substance of abuse or had signs of consumption the day of the interview were excluded. Results: Eighty six percent of respondents had at least one psychiatric disorder. The most common were substance use disorders (76\%). Among these, marijuana dependence disorder had the higher frequency (51\%). Disruptive behavior disorders had also a high prevalence (38\%), followed by anxiety disorders (27\%), attention deficit and hyperactivity disorder (26\%), and affective disorders (21\%). Conclusions: There is a high prevalence of psychiatric morbidity among teenage offenders which could undermine rehabilitation.

(Rev Med Chile 2014; 142: 1377-1384)

Key words: Adolescent; Mental Health; Prevalence.

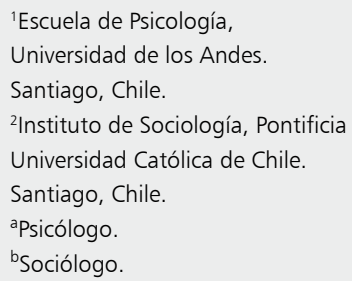

Recibido el 28 de mayo de 2014 aceptado el 22 de octubre de 2014.

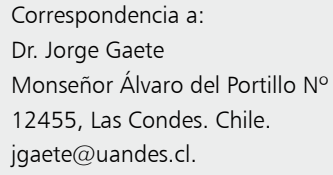

$\mathrm{D}$ esde la entrada en vigencia en Chile de la ley 20.084 en el año 2007, la cual establece un sistema de responsabilidad penal de los adolescentes entre 14 y 18 años, se hace necesario preguntarnos por las características de estos adolescentes, y en particular por su estado de su salud mental. Hay pocos estudios disponibles en Chile que hayan evaluado la presencia de trastornos psiquiátricos en esta población ${ }^{1}$.

En el mundo se ha evidenciado una alta morbilidad psiquiátrica en adolescentes infractores de $l e y^{2-6}$. Uno de los estudios más grandes en Estados Unidos de Norteamérica reunió una muestra de 1.829 adolescentes entre 10 a 18 años, detenidos en el condado de Cook (Illinois). Según este estudio, $66,3 \%$ de los hombres y $73,8 \%$ de las mujeres tuvieron al menos un trastorno psiquiátrico. El trastorno más frecuente fue el trastorno por uso de marihuana (Hombres, 44,8\%; Mujeres, 40,5\%), seguido del trastorno de conducta (Hombres, 37,8\%; Mujeres, 40,6\%). Otros trastornos frecuentes fueron los trastornos ansiosos y los trastornos por uso de alcohol ${ }^{7,8}$. En Inglaterra y Gales, en una muestra de 301 jóvenes infractores de ley, con un promedio de edad de 15,7 años, $31 \%$ presentó alguna patología mental que requería tratamiento, siendo las más frecuentes los trastornos depresivos y problemas 
relacionados con el uso de sustancias de abuso". Un estudio en los Países Bajos recolectó una muestra de 204 niños adolescentes encarcelados entre 12 y 18 años entre los años 1998 y 1999. El 90\% de la muestra mostró tener al menos un trastorno psiquiátrico, siendo los más frecuentes el trastorno disruptivo de la conducta (75\%) y los trastornos asociados al uso de sustancias $(55 \%)^{10}$. Por otro lado, un estudio en China comparó a 236 jóvenes infractores de ley con un grupo control de 88 jóvenes voluntarios seleccionados de forma aleatoria en dos escuelas secundarias. Los resultados revelaron que más de $75 \%$ de los jóvenes infractores de ley tuvieron al menos un trastorno psiquiátrico, siendo el trastorno de conducta y los trastornos asociados al uso de sustancia los más prevalentes ${ }^{11}$.

Finalmente, en un meta-análisis efectuado por Fazel et al. (2008), se revisaron 25 estudios entre enero de 1996 y mayo de 2006 que incluyeron 8 países distintos (15 estudios de Estados Unidos de Norteamérica, 4 del Reino Unido, 1 de Australia, 1 de Rusia, 1 de Holanda, 1 de Dinamarca, 1 de Canadá y 1 de España), y que involucraron un total de 13.778 niños y 2.972 niñas adolescentes (edad promedio de 15,6 años, con un rango de 10 a 19 años). El trastorno más prevalente, tal como en otras investigaciones, fue el trastorno de conducta con $52,8 \%$, tanto en hombres como en mujeres. Por otro lado, $10,6 \%$ de los hombres tenía un diagnóstico de depresión frente a $29,2 \%$ de las mujeres, $11,7 \%$ de los hombres tenía trastorno por déficit atencional en comparación con 18,5\% de las mujeres, y 3,3\% de los hombres tenía un trastorno psicótico frente a 2,7\% de las mujeres. Los trastornos asociados al uso de sustancias fueron excluidos en este meta-análisis por diferencias en la recolección de información ${ }^{12}$.

Hasta el conocimiento de los autores, sólo dos estudios en población chilena de adolescentes infractores de ley han tenido entre sus objetivos el determinar la prevalencia de trastornos psiquiátricos. El estudio desarrollado por la Fundación Tierra de Esperanza (2007) entrevistó a 183 adolescentes que se encontraban cumpliendo condena en régimen cerrado en las regiones Metropolitana, Quinta y Sexta (comunas de San Bernardo, Santiago, Limache y Graneros) ${ }^{13}$. Según este estudio, $62,0 \%$ de la muestra tendría al menos un trastorno de salud mental. Los trastornos más prevalentes fueron los trastornos asociados por uso de sustancias $(38,9 \%)$. Otros trastornos prevalentes fueron los trastornos disruptivos con $41,2 \%$ (trastorno de conducta, $31,7 \%$; trastorno oposicionista desafiante, $13,6 \%$; y trastorno de déficit atencional, 12,2\%). Los trastornos ansiosos se presentaron en 33,0\%. En cuanto a los trastornos afectivos (14,5\%), el más prevalente fue el episodio depresivo mayor con $10,4 \%{ }^{13}$. Finalmente, el estudio de Rioseco et al. (2009) recogió información de 100 adolescentes hombres infractores de ley, comparados con un grupo control pareados según rango de edad y nivel socio-económico, en la provincia de Concepción ${ }^{14}$. La prevalencia de trastornos psiquiátricos en los últimos 12 meses fue de $64,0 \%$. El trastorno de conducta fue el más frecuente $(46,9 \%)$, seguido por los trastornos por consumo de sustancias, en particular el abuso y dependencia a alcohol $(26,0 \%$ y $12,0 \%$, respectivamente) y el abuso y dependencia a marihuana $(10,2 \%$ y $16,3 \%$, respectivamente).

Estas cifras son significativamente más altas que la prevalencia de trastornos psiquiátricos en la población general en Chile, donde sólo 18,2\% de los adolescentes, entre 12 y 18 años, de la Región Metropolitana manifiestan tener al menos un trastorno psiquiátrico que les cause un malestar significativo $^{15}$. Por otro lado, el perfil psicopatológico es diferente al de la población general, donde el trastorno asociado al uso de sustancias es de 3,3\%, y solo 9,9\% tendría un trastorno disruptivo $(5,8 \%$ de TDAH, trastorno de conducta con $3,7 \%$, y $2,7 \%$ con trastorno oposicionista desafiante). Por otro lado, los trastornos ansiosos y depresivos tendrían una prevalencia de $7,9 \%$, y 7,8\%, respectivamente ${ }^{15}$.

En los últimos años se han levantado voces en países desarrollados sobre la urgencia de cubrir las altas necesidades de atención de esta población sobre todo dado que los recursos disponibles son insuficientes ${ }^{16,17}$. Esto ha impulsado a realizar estudios de prevalencia para cuantificar el problema y poder generar conciencia entre las autoridades para el destino de fondos para el tratamiento de estos jóvenes ${ }^{7,12,18,19}$. De esta forma, existe la necesidad en nuestro país de conocer mejor esta problemática.

El objetivo de esta investigación es determinar la prevalencia de trastornos psiquiátricos en hombres adolescentes infractores de ley entre 14 y 17 años, que se encuentran cumpliendo algún tipo de condena. 
Trastornos psiquiátricos en adolescentes infractores de la ley - J. Gaete et al

\section{Sujetos y Método}

El diseño de esta investigación corresponde a un estudio observacional transversal, cuantitativo.

\section{Participantes}

Este estudio se enmarca dentro de un estudio mayor, financiado por Fondecyt (Regular 20122014, No 1121107), cuyo objetivo es determinar el impacto del consumo de sustancias en la carrera delictual de jóvenes infractores de ley cumpliendo alguna condena por el sistema judicial chileno, con un diseño longitudinal. Para este estudio mayor se eligieron centros en los cuales esta población estuviera cursando su condena, por lo que la selección de la muestra fue por conveniencia. La participación en las evaluaciones fue voluntaria y previo consentimiento de los jóvenes. Estos jóvenes se encontraban cumpliendo su condena en centros cerrados (Región Metropolitana [RM], V Región y VI Región), en centros semi-cerrados (RM y V Región), y en programas de libertad asistida simple y libertad asistida especial (RM). El trabajo de campo se realizó durante el segundo semestre de 2012.

Para los propósitos de esta publicación, se presentan los análisis de las entrevistas a adolescentes hombres, infractores de ley con edades entre 14 y 17 años.

\section{Instrumentos}

Las características socio-demográficas fueron recogidas a través de una entrevista estructurada a cargo de sociólogos titulados.

Para determinar la presencia de trastornos psiquiátricos se aplicó la Mini International Neuropsychiatric Interview para Niños y Adolescentes (MINI-Kid), la cual fue originalmente desarrollada para diagnosticar trastornos según el Diagnostic Statistical Manual of Mental Disorders, Fourth Edition (DSM-IV), de forma sencilla y económi$\mathrm{Ca}^{20}$. El MINI-Kid cuenta con estudios de validez y confiabilidad en varios idiomas ${ }^{21}$. Además, este instrumento ha sido utilizado previamente en población adolescente chilena ${ }^{22,23}$. Estas entrevistas fueron realizadas por psicólogos titulados capacitados, lo cual permitió asegurar precisión y confiabilidad diagnóstica.

Sin embargo, debido al tiempo reducido disponible para las evaluaciones, la complejidad en la evaluación de algunos trastornos y su pertinencia en esta población, se excluyeron los siguientes: trastornos bipolares, trastornos de la alimentación, trastornos generalizados del desarrollo, trastornos por tics, y trastornos psicóticos. Por otro lado, se solicitó a los evaluadores que registraran si los entrevistados consumieron sustancias de abuso previo a la entrevista o si bajo su percepción el entrevistado estaba bajo los efectos de una sustancia de abuso.

\section{Análisis estadístico}

Las prevalencias fueron calculadas como porcentajes con $95 \%$ de intervalos de confianza, con el software STATA versión 12. Además, para los propósitos del análisis, se excluyeron los casos que durante la entrevista manifestaron consumo de sustancias el día de la entrevista, o que tuvieran apariencia de haber consumido $(\mathrm{n}=23,5,04 \%)$. Finalmente, para este estudio sólo se presentan los diagnósticos actuales presentes en esta población. Para el caso de los trastornos por consumo de sustancias, dependencia y abuso, se hicieron análisis por separado para evaluar la prevalencia de estos, y también se unieron ambos trastornos por tipo de sustancia para evaluar la comorbilidad de más de una sustancia y la correlación de ellas.

El estudio de correlaciones de cada par de variables presentado en este estudio, se realizó para todas las observaciones que tuvieron información válida de cada variable (pairwise deletion method), usando como nivel de significancia un valor $\mathrm{p} \leq 0,05$.

\section{Consideraciones éticas}

El estudio fue aprobado por el comité de ética del Instituto de Sociología de la Pontificia Universidad Católica de Chile.

\section{Resultados}

\section{Descripción de la muestra}

Un total de 489 jóvenes, entre 14 y 17 años, participaron en este estudio. La edad media fue de 16,4 (95\% IC: 16,3-16,5). La mayoría de estos jóvenes había alcanzado sólo estudios de enseñanza básica al momento de la entrevista $(70,1 \%)$. Uno de cada 5 adolescentes había trabajado alguna vez antes del estudio y $10 \%$ refería tener hijos. El 14,3\% había vivido en alguna residencia de menores, y 6,5\% refirió haber vivido en la calle 
alguna vez antes de la entrevista. Finalmente, cerca de un tercio de los jóvenes refería haber tenido a su padre en la cárcel, $11,5 \%$, a su madre y $23,7 \%$ a un hermano (Tabla 1).

\section{Prevalencias de trastornos psiquiátricos}

De los 489 casos con información completa, se pudo apreciar una prevalencia de $86,3 \%$ de cualquier trastorno psiquiátrico. Entre estos, los más frecuentes fueron aquellos trastornos asociados al consumo de sustancias de abuso con $75,5 \%$. Los trastornos disruptivos serían el segundo grupo de trastornos psiquiátricos más frecuente, con $37,8 \%$ (trastorno de conducta, 27,7\%; y el trastorno oposicionista desafiante, 27,1\%). El tercer trastorno más prevalente fue el trastorno por déficit atencional con $25,4 \%$, siendo el tipo combinado el más frecuente. el episodio depresivo mayor tuvo

Tabla 1. Descripción de la muestra

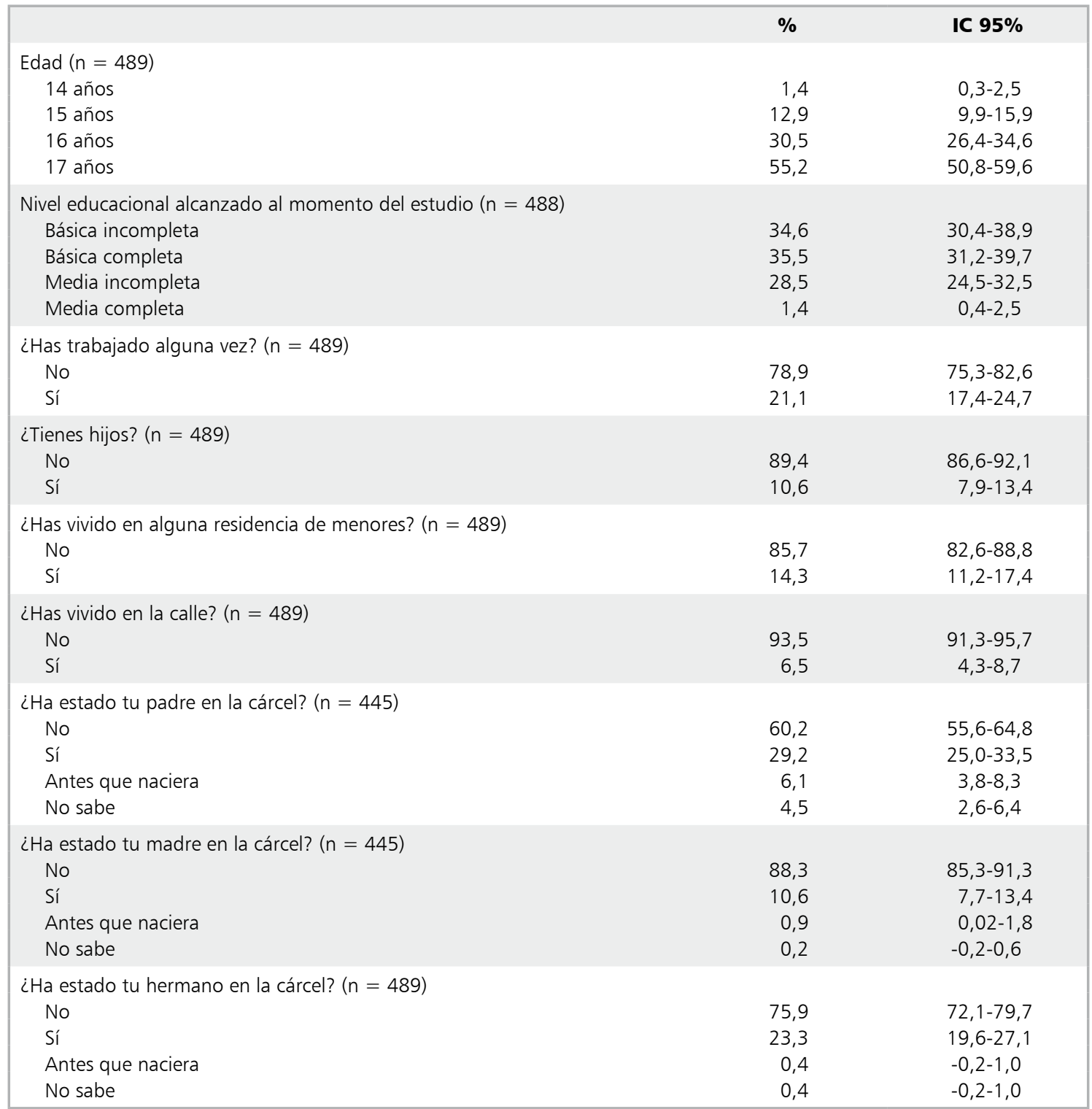


una prevalencia de 19,7\%. Los trastornos ansiosos evaluados tuvieron una prevalencia de $2,1 \%$ (para fobia social) a 8,5\% (para agorafobia) (tabla 2).

La dependencia a marihuana fue el trastorno más prevalente dentro de este grupo, con $51 \%$, seguido de la dependencia a alcohol, con 26,8\%. en tercer lugar encontramos la dependencia a Nicotina (21,8\%), seguida de la dependencia a Cocaína, con 19,2\% (Tabla 3 ).

\section{Comorbilidad de trastornos psiquiátricos}

La comorbilidad psiquiátrica fue altamente prevalente, $67,7 \%$ de los entrevistados reportaron 2 o más trastornos comórbidos. Por ejemplo, $16,4 \%$ de los adolescentes tuvo 2 trastornos psiquiátricos en el mismo período de tiempo (Tabla 4). Por otro lado, $47,4 \%$ de los adolescentes reportó abuso o dependencia a al menos 2 sustancias distintas (Tabla 5). En Tabla 6 se muestra la correlación de los trastornos por uso de sustancias.

Tabla 2. Prevalencia de trastornos psiquiátricos

\begin{tabular}{|lrc|}
\hline Trastorno psiquiátrico & \% & 95\% IC \\
\hline Cualquier trastorno psiquiátrico & 86,3 & $83,2-89,4$ \\
\hline Cualquier trastorno por & & \\
consumo de sustancias & 75,5 & $71,6-79,3$ \\
\hline Trastornos afectivos* & 20,9 & $17,3-24,6$ \\
Episodio depresivo mayor & 19,7 & $16,1-23,3$ \\
Distímia & 1,3 & $0,3-2,3$ \\
\hline Trastornos ansiosos & 27,1 & $23,0-31,1$ \\
Fobia social & 2,1 & $0,8-3,4$ \\
Fobia especifica & 4,7 & $2,7-6,6$ \\
Ansiedad generalizada & 4,9 & $2,9-6,8$ \\
Ansiedad por separación & 4,0 & $2,2-5,8$ \\
Trastorno de pánico & 5,1 & $3,1-7,1$ \\
Agorafobia & 8,5 & $5,9-11,0$ \\
Obsesivo-compulsivo & 5,3 & $3,3-7,3$ \\
Estrés post-traumático & 2,3 & $0,9-3,7$ \\
\hline Trastornos disruptivos ${ }^{\dagger}$ & 37,8 & $33,5-42,2$ \\
Trastorno de conducta & 27,7 & $23,6-31,7$ \\
Oposicionista desafiante & 27,1 & $23,0-31,7$ \\
\hline Trastorno por déficit atencional & 25,6 & $21,6-29,5$ \\
Predominio hiperactividad & 8,9 & $6,3-11,5$ \\
Predominio desatención & 5,3 & $3,3-7,3$ \\
Combinado & 11,4 & $8,5-14,3$ \\
\hline Na & & \\
\hline
\end{tabular}

Notas: *Trastornos afectivos no incluyen al trastorno bipolar ni episodios (hipo) maniacos. ${ }^{\dagger}$ Se consideran trastorno de conducta y trastorno oposicionista desafiante con posibilidad de ser comórbidos.
Tabla 3. Prevalencia de trastornos asociados al uso de sustancias

\begin{tabular}{|lcc|}
\hline $\begin{array}{l}\text { Trastorno asociado al uso de } \\
\text { sustancias }\end{array}$ & $\%$ & $\mathbf{9 5 \%}$ IC \\
\hline Trastornos por uso de alcohol & 31,7 & $27,5-35,9$ \\
$\quad$ Dependencia de alcohol & 26,8 & $22,8-30,9$ \\
$\quad$ Abuso de alcohol & 4,9 & $2,9-6,8$ \\
Trastornos por uso de marihuana & 64,7 & $60,2-68,8$ \\
$\quad$ Dependencia de marihuana & 51,0 & $46,4-55,5$ \\
Abuso de marihuana & 13,7 & $10,6-16,9$ \\
Trastornos por uso de nicotina & 27,3 & $23,3-31,3$ \\
$\quad$ Dependencia de nicotina & 21,8 & $18,0-25,5$ \\
Abuso de nicotina & 5,5 & $3,4-7,6$ \\
Trastornos por uso de cocaína & 21,3 & $17,5-25,0$ \\
$\quad$ Dependencia de cocaína & 19,2 & $15,7-22,8$ \\
Abuso de cocaína & 2,1 & $0,8-3,4$ \\
Trastornos por uso de tranquilizantes & 16,7 & $13,2-20,0$ \\
$\quad$ Dependencia de tranquilizantes & 14,2 & $11,0-17,3$ \\
Abuso de tranquilizantes & 2,5 & $1,1-4,0$ \\
\hline Trastornos por uso de estimulantes & 4,0 & $2,2-5,8$ \\
$\quad$ Dependencia de estimulantes & 3,2 & $1,6-4,8$ \\
Abuso de estimulantes & 0,8 & $0,0-1,7$ \\
\hline
\end{tabular}

Tabla 4. Comorbilidad de trastornos psiquiátricos

\begin{tabular}{|lcc|}
\hline $\begin{array}{l}\text { Número de trastornos } \\
\text { comórbidos }\end{array}$ & $\mathbf{\%}$ & $\mathbf{9 5 \%}$ IC \\
\hline $\begin{array}{l}\text { Ningún trastorno psiquiátrico } \\
1 \text { trastorno psiquiátrico }\end{array}$ & 13,7 & $10,6-16,8$ \\
\hline 2 trastornos psiquiátricos comórbidos & 16,4 & $13,1-19,7$ \\
\hline 3 trastornos psiquiátricos comórbidos & 15,1 & $11,9-18,3$ \\
\hline 4 trastornos psiquiátricos comórbidos & 14,3 & $11,2-17,4$ \\
\hline
\end{tabular}

Tabla 5. Comorbilidad de trastornos asociados al uso de sustancias

\begin{tabular}{|lcc|}
\hline & \% & $\mathbf{9 5 \%}$ IC \\
\hline $\begin{array}{l}\text { Sin trastornos asociado al uso de } \\
\text { sustancias }\end{array}$ & 24,5 & $20,7-28,4$ \\
$\begin{array}{l}\text { Trastornos por uso de 1 sustancia } \\
\text { de abuso }\end{array}$ & 29,0 & $25,0-33,1$ \\
$\begin{array}{l}\text { Trastornos por uso de } 2 \text { sustancia } \\
\text { de abuso }\end{array}$ & 21,1 & $17,4-24,7$ \\
$\begin{array}{l}\text { Trastornos por uso de } 3 \text { sustancia } \\
\text { de abuso }\end{array}$ & 15,1 & $11,9-18,3$ \\
\hline
\end{tabular}


Tabla 6. Correlación (r) de trastornos por uso de sustancias

\begin{tabular}{|c|c|c|c|c|c|c|c|}
\hline $\begin{array}{l}\text { Trastorno, } \\
\text { según la } \\
\text { sustancia }\end{array}$ & Alcohol & Marihuana & Cocaína & Nicotina & Tranquilizantes & Estimulantes & Inhalantes \\
\hline Alcohol & 1 & & & & & & \\
\hline \multirow[t]{2}{*}{ Marihuana } & 0,2510 & 1 & & & & & \\
\hline & 0,0000 & & & & & & \\
\hline \multirow[t]{2}{*}{ Cocaína } & 0,2866 & 0,2347 & 1 & & & & \\
\hline & 0,0000 & 0,0000 & & & & & \\
\hline \multirow[t]{2}{*}{ Nicotina } & 0,1090 & 0,1789 & 0,0394 & 1 & & & \\
\hline & 0,0173 & 0,0001 & 0,3911 & & & & \\
\hline \multirow[t]{2}{*}{ Tranquilizantes } & 0,2055 & 0,2838 & 0,3209 & 0,0307 & 1 & & \\
\hline & 0,0000 & 0,0000 & 0,0000 & 0,5037 & & & \\
\hline \multirow[t]{2}{*}{ Estimulantes } & 0,0685 & 0,1289 & 0,2354 & $-0,0046$ & 0,2840 & 1 & \\
\hline & 0,1353 & 0,0049 & 0,0000 & 0,9211 & 0,0000 & & \\
\hline \multirow[t]{2}{*}{ Inhalantes } & 0,0673 & 0,0340 & 0,0884 & 0,0749 & 0,1029 & $-0,0094$ & 1 \\
\hline & 0,1425 & 0,4587 & 0,0539 & 0,1029 & 0,0248 & 0,8387 & \\
\hline
\end{tabular}

Nota: Recuadros sombreadas resaltan las correlaciones significativas con un valor $p<0,05$.

Varias de estas correlaciones fueron significativas a un $\mathrm{p}<0,05$, es decir, el uso de una sustancia está relacionado con el uso de otras al mismo tiempo. En particular, las correlaciones más altas fueron entre trastorno por uso de cocaína y de tranquilizantes $(r=0,3209 ; \mathrm{p}=0,0000)$, seguido por trastorno por uso de alcohol y cocaína $(\mathrm{r}=0,2866$; $\mathrm{p}=0,0000)$.

\section{Discusión}

Nuestros resultados muestran una realidad preocupante: más de $85 \%$ de los adolescentes que se encuentran cumpliendo condena tiene al menos un diagnóstico psiquiátrico. Los grupos de trastornos más prevalente fueron aquellos asociados al consumo de sustancias de abuso y los trastornos disruptivos. Ambos resultados son confirmados por la literatura revisada $a^{3,7,13,14,24}$. Algunas diferencias entre los estudios pueden explicarse principalmente por dos razones: i) uso de distintos instrumentos diagnósticos, y ii) la muestra analizada en este estudio incluyó a sujetos cumpliendo condena en recintos semicerrados y abiertos, población que no siempre ha sido incluida en otros trabajos. El trastorno de déficit atencional fue de $25,4 \%$, similar a otro estudio $^{6}$, pero relativamente más alto que en otros estudios revisados ${ }^{7,13,18,24}$. También pueden existir diferencias en las muestras usadas en los distintos estudios. Los trastornos afectivos y ansiosos también muestran una prevalencia concordante con otros estudios ${ }^{6,24}$.

Existe una alta comorbilidad de trastornos psiquiátricos en esta población, donde $67,7 \%$ de la muestra posee dos o más trastornos. Esto sería consistente con otros estudios revisados ${ }^{3,10}$. De la misma manera, también existe una alta comorbilidad en trastornos relacionados al uso de sustancias, donde $47,4 \%$ de los individuos evaluados tienen dependencia o abuso a dos o más sustancias, lo que coincide con otras investigaciones ${ }^{24}$.

\section{Limitaciones}

El diagnóstico psiquiátrico es una acción difícil de realizar, especialmente en la población infantojuvenil, donde es necesaria la información que puedan proporcionar otros actores importantes en la vida de los sujetos, la cual estuvo ausente en este estudio. También es importante descartar que la patología psiquiátrica no se deba a otras causas 
médicas o por el mismo uso de sustancias de abuso. Por lo anterior, los diagnósticos en este estudio tienen carácter provisorio y deben ser contrastados con evaluaciones futuras más completas.

La MINI-Kid es relativamente breve, lo cual permitió llevar a cabo este estudio y facilitó la participación de los sujetos; sin embargo, los trastornos que se pueden evaluar tienen distintos niveles de complejidad y se requiere de un buen conocimiento en psicopatología de parte de los entrevistadores. Debido a la necesidad de alta experiencia clínica, trastornos como el trastorno bipolar o trastornos psicóticos fueron excluidos. Así mismo, se excluyó la evaluación de otros trastornos que parecieron menos relevantes para esta población (por ejemplo, trastornos de la alimentación, más prevalente en mujeres). Lo anterior limitó la gama de trastornos evaluados.

La exclusión de sujetos con consumo de sustancias o apariencia de este el día de la entrevista permitió ganar en confiabilidad de los análisis. Sin embargo, no podemos excluir que no hayan habido otros sujetos cuyas respuestas hayan sido afectadas por el uso de alguna sustancia psicoactiva, pero que no lo hayan reportado, o no haya sido evidente para los entrevistadores. Una solución posible a esta problemática podría haber sido el examen biológico de la presencia de alguna sustancia durante la entrevista, pero esta solución escapaba a los recursos disponibles. Al mismo tiempo, esta exclusión en los análisis, en un pequeño grado, podría estar disminuyendo la prevalencia de trastornos asociados al uso de sustancias en esta población.

\section{Conclusiones}

La población estudiada presenta una alta prevalencia de patología psiquiátrica que podría obstaculizar el uso de recursos y capacidades personales en el proceso de rehabilitación de estos jóvenes, el que por sí mismo ya es difícil. Es urgente que como sociedad, y en particular, las instituciones relacionadas al cuidado de estos jóvenes, dispongan de los recursos económicos y profesionales que permitan asegurar una ayuda efectiva en el proceso de rehabilitación. Esto debiera incluir la evaluación diagnóstica y tratamiento de la patología psiquiátrica.

Esta es una población en la que co-ocurren el consumo de sustancias de abuso y patología psiquiátrica, condiciones difíciles de tratar; por lo que los esfuerzos deben estar centrados en la realización de ensayos clínicos aleatorios que permitan extraer información validada científicamente. Una reciente revisión muestra que existen muy pocos estudios de evaluación de la efectividad de intervenciones para esta población, con resultados poco concluyentes ${ }^{25}$; por lo que los esfuerzos de grupos de investigación a nivel nacional podrían tener un impacto más allá de nuestra realidad local. Entre las intervenciones que se podrían proponer, está la de generar instancias formales de formación de profesionales de salud mental especializados en este tipo de poblaciones asociadas a centros académicos. Por ejemplo, es posible ver en países como Estados Unidos de Norteamérica e Inglaterra la existencia de subespecializaciones en Psiquiatría para este tipo de poblaciones. Por otro lado, se podrían evaluar intervenciones que incorporen distintos niveles de gravedad y ajustar los recursos a cada nivel, con continuidad de los apoyos más allá de los períodos de condena. Además, se podría proponer, dentro de los centros abiertos, que los jóvenes participen en intervenciones terapéuticas como parte de las condiciones de la condena.

Finalmente, entre las preguntas a contestar en análisis futuros están el conocer mejor los factores que pueden afectar la expresión de estos trastornos.

\section{Referencias}

1. Maldonado F. Prevalencia de patologías de salud mental en la población adolescente privada de libertad: experiencias nacionales y comparadas. Revista Ius et Praxis 2013; (1): 329-62.

2. Abram KM, Teplin LA, McClelland GM, Dulcan MK. Comorbid psychiatric disorders in youth in juvenile detention. Archives of general psychiatry 2003; 60 (11): 1097-108.

3. Washburn JJ, Teplin LA, Voss LS, Simon CD, Abram KM, McClelland GM. Psychiatric disorders among detained youths: a comparison of youths processed in juvenile court and adult criminal court. Psychiatric services 2008; 59 (9): 965-73.

4. Rogers KM, Pumariega AJ, Atkins DL, Cuffe SP. Conditions associated with identification of mentally ill youths in juvenile detention. Community mental health journal 2006; 42 (1): 25-40.

5. Wasserman GA, McReynolds LS, Lucas CP, Fisher P, 
Santos L. The voice DISC-IV with incarcerated male youths: prevalence of disorder. Journal of the American Academy of Child and Adolescent Psychiatry 2002; 41 (3): 314-21.

6. Odgers CL, Burnette ML, Chauhan P, Moretti MM, Reppucci ND. Misdiagnosing the problem: mental health profiles of incarcerated juveniles. The Canadian child and adolescent psychiatry review $=$ La revue canadienne de psychiatrie de l'enfant et de l'adolescent 2005; 14 (1): 26-9.

7. Teplin LA, Abram KM, McClelland GM, Dulcan MK, Mericle AA. Psychiatric disorders in youth in juvenile detention. Archives of general psychiatry 2002; 59 (12): 1133-43.

8. Teplin LA, Abram KM, McClelland GM, Mericle AA, Dulcan MK, Washburn JJ. Psychiatric Disorders of Youth in Detention 2006 Abril 30, 2014. Available from: https:// http://www.ncjrs.gov/pdffiles1/ojjdp/210331.pdf.

9. Chitsabesan P, Kroll L, Bailey S, Kenning C, Sneider S, Macdonald W, et al. Mental health needs of young offenders in custody and in the community. The British Journal of Psychiatry 2006; 188 (6): 534-40.

10. Vreugdenhil C, Doreleijers TA, Vermeiren R, Wouters LF, van den Brink W. Psychiatric disorders in a representative sample of incarcerated boys in the Netherlands. Journal of the American Academy of Child and Adolescent Psychiatry 2004; 43 (1): 97-104.

11. Zhou J, Cai W, Chen C, Wang H, Zhang S, Luo Y, et al. Prevalence of mental disorders in the male juvenile detention centers of Hunan and Sichuan. Zhong nan da xue xue bao Yi xue ban = Journal of Central South University Medical sciences 2012; 37 (3): 217-21.

12. Fazel S, Doll H, Langstrom N. Mental disorders among adolescents in juvenile detention and correctional facilities: a systematic review and metaregression analysis of 25 surveys. Journal of the American Academy of Child and Adolescent Psychiatry 2008; 47 (9): 1010-9.

13. Fundación Tierra Esperanza. Estudio Diagnóstico de la Situación Actual de la Atención en Salud Mental y Psiquiatría para la Población de Adolescentes que Cumplen Condena en CIP-CRC. Santiago, Chile: 2007.

14. Rioseco P, Vicente B, Saldivia S, Cova F, Melipillán R, Rubi P. Prevalencia de trastornos psiquiátricos en adolescentes infractores de ley: Estudio caso-control. Rev Chil Neuro-Psiquiatr 2009; 47: 190-200.
15. Vicente B, de la Barra F, Saldivia S, Kohn R, Rioseco P, Melipillán R. Prevalence of child and adolescent psychiatric disorders in Santiago, Chile: a community epidemiological study. Social psychiatry and psychiatric epidemiology 2012; 47 (7): 1099-109.

16. Grisso T. Adolescent offenders with mental disorders. The Future of children/Center for the Future of Children, the David and Lucile Packard Foundation 2008; 18 (2): 143-64.

17. MacReady N. US faces crisis in mental health care for juvenile offenders. Lancet 2009; 374 (9690): 601.

18. Penner EK, Roesch R, Viljoen JL. Young Offenders in Custody: An International Comparison of Mental Health Services. International Journal of Forensic Mental Health 2011; 10 (3): 215-32.

19. Douglas NPE. A Health Needs Assessment for Young Women in Young Offender Institutions. Youth Justice Board for England and Wales, 2006.

20. Sheehan DV, Lecrubier Y, Sheehan KH, Amorim P, Janavs J, Weiller E, et al. The Mini-International Neuropsychiatric Interview (M.I.N.I.): the development and validation of a structured diagnostic psychiatric interview for DSM-IV and ICD-10. The Journal of clinical psychiatry 1998; 59 Suppl 20: 22-33; quiz 4-57.

21. Sheehan DV, Sheehan KH, Shytle RD, Janavs J, Bannon Y, Rogers JE, et al. Reliability and validity of the Mini International Neuropsychiatric Interview for Children and Adolescents (MINI-KID). The Journal of clinical psychiatry 2010; 71 (3): 313-26.

22. Araya R, Montero-Marín J, Barroilhet S, Fritsch R, Gaete J, Montgomery A. Detecting depression among adolescents in Santiago, Chile: sex differences. BMC psychiatry 2013; 13: 122.

23. De la Peña FEG, Pérez A, Palacios L. Validación Concurrente para Trastornos Externalizados del MINI-Kid y la Entrevista Semi-estructurada para Adolescentes. Revista Chilena de Psiquiatría y Neurología de la Infancia y Adolescencia 2009; 20 (1): 8-12.

24. SUSTENTANK. Estudio de Prevalencia de Trastornos Mentales en Adolescentes Privados de Libertad en Virtud de la Ley $N^{\circ}$ 20.084. Santiago, Chile: 2012.

25. Perry AE, Neilson M, Martyn-St James M, Glanville JM, McCool R, Duffy S, et al. Interventions for drug-using offenders with co-occurring mental illness. The Cochrane database of systematic reviews 2014; 1: CD010901. 